\title{
Finite-temperature Gutzwiller projection for strongly correlated electron systems
}

\author{
Wan-Sheng Wang, Xiang-Mei He, Da Wang, and Qiang-Hua Wang* \\ National Laboratory of Solid State Microstructures and Department of Physics, Nanjing University, Nanjing 210093, China
}

\author{
Z. D. Wang and F. C. Zhang \\ Department of Physics, Center of Theoretical and Computational Physics, University of Hong Kong, Pokfulam Road, Hong Kong, China
}

(Received 19 May 2010; revised manuscript received 28 June 2010; published 7 September 2010)

\begin{abstract}
We generalized the Gutzwiller projectional variational method for the ground state of strongly correlated electron systems to the case of finite temperature. Under the Gutzwiller approximation, we show that this maps to a finite temperature renormalized mean-field theory. As one of the key ingredients in the theory, we obtained an explicit expression of the projection entropy or the entropy change due to the projection. We illustrate the application of the theory to the Anderson impurity problem and the half-filled Hubbard model and compare the theory to more elaborate techniques. We find qualitative agreement. The theory can be applied to a wide variety of Hubbard, $t-J$, and Anderson impurity models.
\end{abstract}

DOI: 10.1103/PhysRevB.82.125105

PACS number(s): 71.27.+a, 71.10.Fd, 71.30. $+\mathrm{h}$

\section{INTRODUCTION}

The Hubbard model and its descendant $t$ - $J$ model describe electronic systems with strong but short-range Coulomb interactions. These models are widely used to describe strongly correlated electrons, especially those originated from $d$ or $f$ orbitals. However, these models are hard to treat reliably except in one dimension. An important effect of strong correlations is the narrowing of the energy band, apart from the development of Hubbard bands and spin correlations. Traditional mean-field theories fail to account for the band narrowing and therefore fail to capture the essential physics in strongly correlated systems. The variational Gutzwiller projection is satisfying in this respect, at least qualitatively. ${ }^{1}$ In such an approach, one tries to find the variational ground state $|G\rangle=P\left|G_{0}\right\rangle$, where $\left|G_{0}\right\rangle$ is a suitable state of free particles and the Gutzwiller projection operator $P=\Pi_{i}\left[1-\left(1-g_{i}\right) n_{i \uparrow} n_{i \downarrow}\right]$ accounts for the correlation effect by reweighting the configurations with double occupancies in $\left|G_{0}\right\rangle$. The variational parameters are $\left\{g_{i}\right\}$ and the bare order parameters encoded in $\left|G_{0}\right\rangle$. If $g_{i}=0$ the projection forbids any double occupancy, and therefore a half-filled Hubbard model becomes a Mott insulator since charge fluctuations are frozen. It has been established that under the so-called Gutzwiller approximation the variational theory leads to a renormalized mean-field theory (RMFT) (Refs. 2 and 3) with an effective Hamiltonian $H_{0}$ that describes free particles whose ground state is $\left|G_{0}\right\rangle$. This theory can then be used to calculate the energy $E=\langle G|H| G\rangle /\langle G \mid G\rangle$ and also the coherent part of the quasiparticle excitation spectra in the original system under concern. The results ${ }^{4}$ compare fairly well with those obtained by Gutzwiller projection variational Monte Carlo (VMC) calculations. ${ }^{5}$

The success of Gutzwiller projection method for the ground state motivates us to ask whether it could be extended to finite temperatures. In the latter cases, one has to deal with ensemble averages instead of averaging in a pure state. Therefore it seems technically unlikely that VMC can be conveniently performed at finite temperatures. Since RMFT qualitatively reproduces VMC results, we take the alternative to develop a finite-temperature version of RMFT, which we call TRMFT.
In this work, we generalize the Gutzwiller projectional variational method for the ground state to finite temperatures. For this purpose, we find the free energy as a functional of the projection parameters as well as the order parameters under the Gutzwiller approximation. The free-energy functional contains two parts. One is the internal-energy functional, which turns out to be similar to the zero-temperature case. The other part is related to the entropy functional, which is highly nontrivial to evaluate. We find that it can be decomposed into a free-fermion entropy and a projection entropy or the entropy change due to projection. The latter plays a key role at finite temperatures. Finally the optimization of the free-energy functional leads to the TRMFT we are after. We illustrate the application of the theory to the Anderson impurity problem and the half-filled Hubbard model and find qualitative agreements in comparison to results from more elaborate numerical renormalization group (NRG) and dynamical MFT (DMFT) techniques. We conclude that the TRMFT can be applied to a variety of Hubbard, $t-J$, and Anderson impurity models.

The rest of the paper is structured as follows. In Sec. II we derive the internal energy and the entropy of the system at finite temperatures using a variational projected density matrix, which then enables us to construct the free energy, the optimization of which leads to the TRMFT. In Sec. III the theory is applied to the $t$ - $J$ model and compared with the associated gauge theory. In Sec. IV we apply the theory to the Anderson model and compare to the corresponding NRG results. In Sec. V the theory is applied to the half-filled Hubbard model. We discuss the phase diagram in the interactiontemperature space and compare the result to that from DMFT. Section VI is a summary of this work.

\section{DEVELOPMENT OF TRMFT}

For general purposes, we consider the so-called $t-U-J$ model, ${ }^{6}$

$$
H=-t \sum_{\langle i j\rangle \sigma}\left(c_{i \sigma}^{\dagger} c_{j \sigma}+\text { H.c. }\right)+J \sum_{\langle i j\rangle} \mathbf{S}_{i} \cdot \mathbf{S}_{j}+U \sum_{i} n_{i \uparrow} n_{i \downarrow} .
$$

As an effective model, it is assumed that the spin exchange $J$ and the on-site repulsion $U$ are independent parameters at 
our disposal. In the limit of $U \rightarrow \infty$ (so that no double occupancy is allowed) the standard $t-J$ model is recovered whereas in the limit of $J \rightarrow 0$ the standard Hubbard model is obtained. In fact, it is believed that adding the $J$ term to the standard Hubbard model improves the accounting of the effect of spin exchange and adding the $U$ term in the standard $t-J$ model and relaxing the no-double-occupancy condition improve the accounting of the effect of charge fluctuations in approximate treatments, although these are unnecessary in exact treatments that are still lacking.

At finite temperatures, the desired variational theory should minimize the free energy. For this purpose, we seek a variational density matrix $\rho=P \rho_{0} P$ in the canonical ensemble or $\tilde{\rho}=\mathcal{P} \widetilde{\rho}_{0} \mathcal{P}$ in the grand-canonical ensemble, where $\rho_{0}=e^{-\beta H_{0}}$ and $\tilde{\rho}_{0}=e^{\left[-\beta\left(H_{0}-\mu_{V} N_{e}\right)\right]}$ describe a free particle system governed by the Hamiltonian $H_{0}$ that we are looking for and $\mathcal{P}$ is the projection operator to be specified in the grandcanonical ensemble. Here $N_{e}$ is the number of electrons and $\mu_{V}$ is the variational chemical potential. For reasons that will become clear below, we will use the grand-canonical and canonical ensemble density matrices interchangeably, but eventually we obtain the Hemholtz free energy $F=E-T S$ at a given temperature and electron number, where $E$ and $S$ are the internal energy and the entropy, respectively. By Peierls theorem, ${ }^{7}$ the variational free energy $F$ should satisfy the inequality $F \geq F$ exact.

\section{A. Internal energy}

The internal energy $E$ is most conveniently evaluated in the grand-canonical ensemble. In this case, the projection operator should be modified in such a way that it does not change the electron-density distribution, namely, $f_{i}=\left\langle n_{i}\right\rangle_{0}=\left\langle n_{i}\right\rangle$. Henceforth we use the short-hand notations: $\langle O\rangle=\operatorname{Tr} \tilde{\rho} O / \tilde{Z}$ and $\langle O\rangle_{0}=\operatorname{Tr} \tilde{\rho}_{0} O / \widetilde{Z}_{0}$ for any operator $O$, where $\widetilde{Z}_{0}=\operatorname{Tr} \tilde{\rho}_{0}$ and $\widetilde{Z}=\operatorname{Tr} \tilde{\rho}=\widetilde{Z}_{0}\left\langle\mathcal{P}^{2}\right\rangle_{0}$. The requirement is achieved by introducing fugacity factors in the projection operator, ${ }^{3,8-10}$ which we write as

$$
\mathcal{P}=\Pi_{i}\left(E_{i}+y_{i} Q_{i}+y_{i}^{2} g_{i} D_{i}\right)
$$

in which

$$
\begin{gathered}
E_{i}=\left(1-n_{i \uparrow}\right)\left(1-n_{i \downarrow}\right), \\
Q_{i}=n_{i \uparrow}\left(1-n_{i \downarrow}\right)+n_{i \downarrow}\left(1-n_{i \uparrow}\right), \\
D_{i}=n_{i \uparrow} n_{i \downarrow}
\end{gathered}
$$

are empty, single, and double occupation operators at site $i$, respectively, and $y_{i}$ and $g_{i}$ control the average electron number and double occupation at site $i$, respectively.

Since $\widetilde{\rho}_{0}$ describes free particles, $\left\langle\mathcal{P}^{2}\right\rangle_{0}$ can be factorized using the finite-temperature version of the Wick's theorem. Under the Gutzwiller approximation, only sitewise factorization is retained, $\left\langle\mathcal{P}^{2}\right\rangle_{0}=\Pi_{i} \tilde{z}_{i}$, with

$$
\widetilde{z_{i}}=e_{i 0}+y_{i}^{2} q_{i 0}+y_{i}^{4} g_{i}^{2} d_{i 0} .
$$

Here

$$
e_{i 0}=\left\langle E_{i}\right\rangle_{0}
$$

$$
\begin{aligned}
& q_{i 0}=\left\langle Q_{i}\right\rangle_{0}, \\
& d_{i 0}=\left\langle D_{i}\right\rangle_{0} .
\end{aligned}
$$

We note that the Gutzwiller approximation becomes exact in infinite dimensions and is anticipated to capture the leadingorder effects at finite dimensions. The corresponding quantities after the projection are

$$
\begin{gathered}
e_{i}=\left\langle E_{i}\right\rangle=e_{i 0} / \widetilde{z_{i}}, \\
q_{i}=\left\langle Q_{i}\right\rangle=y_{i}^{2} q_{i 0} / \widetilde{z_{i}}, \\
d_{i}=\left\langle D_{i}\right\rangle=y_{i}^{4} g_{i}^{2} d_{i 0} / \widetilde{z_{i}} .
\end{gathered}
$$

Apart from the obvious normalization condition

$$
e_{i 0}+q_{i 0}+d_{i 0}=e_{i}+q_{i}+d_{i}=1,
$$

we have

$$
e_{i} d_{i} / q_{i}^{2}=g_{i}^{2} e_{i 0} d_{i 0} / q_{i 0}^{2} .
$$

We also require that the electron occupation $f_{i}$ at any site $i$ is not changed by the projection or

$$
f_{i}=q_{i 0}+2 d_{i 0}=q_{i}+2 d_{i} .
$$

By tuning the fugacity factor $y_{i}$, this is always possible. As a result $\left\{e_{i}, q_{i}, d_{i}\right\}$ is uniquely determined by the three conditions stated above and can be related to $\left\{r_{i \sigma}, g_{i}\right\}$, where we defined $r_{i \sigma}=\left\langle n_{i \sigma}\right\rangle_{0}$, with which we have

$$
\begin{gathered}
f_{i}=r_{i \uparrow}+r_{i \downarrow}, \\
e_{i 0}=\left(1-r_{i \uparrow}\right)\left(1-r_{i \downarrow}\right), \\
q_{i 0}=f_{i}-2 r_{i \uparrow} r_{i \downarrow}, \\
d_{i 0}=r_{i \uparrow} r_{i \downarrow} .
\end{gathered}
$$

We assumed that there is no on-site pairing order, which is suitable for the case of repulsive on-site interaction.

It is possible at this stage to state clearly the variational parameter space. In order to specify $\tilde{\rho}_{0}$, we need the singleparticle orbital function $\psi_{n}$ and its fermionic occupation probability $f_{n}$. This should also determine $\left\{r_{i \sigma}\right\}$. Therefore, the variational quantities are $\left\{\psi_{n}, f_{n}, g_{i}\right\}$, which completely specify $\tilde{\rho}$ (and also $\rho$ ). The free energy is understood as a functional of these variables. We shall work out more practical variational parameters in the following development.

With the help of finite temperature Wick's theorem and the Gutzwiller approximation, the internal energy can be obtained in a similar fashion to the case of zero temperature. ${ }^{3}$ First, consider the spin-exchange term. Since $Q_{i} S_{i} Q_{i}=S_{i}$, we have

$$
\left\langle S_{i} \cdot S_{j}\right\rangle=\left\langle S_{i} \cdot S_{j}\right\rangle_{0} y_{i}^{2} y_{j}^{2} / \widetilde{z_{i}} \widetilde{z_{j}}=g_{s}(i) g_{s}(j)\left\langle S_{i} \cdot S_{j}\right\rangle_{0},
$$

where

$$
g_{s}(i)=y_{i}^{2} / \widetilde{z_{i}}=q_{i} / q_{i 0}
$$

is the renormalization factor for the spin moment. The meanfield decoupling of spin exchange is standard 


$$
\left\langle S_{i} \cdot S_{j}\right\rangle_{0}=-(3 / 8)\left(\left|\Delta_{i j}\right|^{2}+\left|\chi_{i j}\right|^{2}\right)+m_{i} m_{j},
$$

where we defined the order parameters

$$
\begin{gathered}
\Delta_{i j}=\left\langle c_{i \downarrow} c_{j \uparrow}-c_{i \uparrow} c_{j \downarrow}\right\rangle_{0}, \\
\chi_{i j}=\sum_{\sigma}\left\langle c_{i \sigma}^{\dagger} c_{j \sigma}\right\rangle_{0}, \\
m_{i}=\sum_{\sigma} \sigma\left\langle c_{i \sigma}^{\dagger} c_{i \sigma}\right\rangle_{0} / 2=\left(r_{i \uparrow}-r_{i \downarrow}\right) / 2 .
\end{gathered}
$$

Second, consider the hopping term. Since

$$
P_{i} c_{i \sigma} P_{i}=c_{i \sigma}\left[\left(1-n_{i \bar{\sigma}}\right) y_{i}+n_{i \bar{\sigma}} y_{i}^{3} g_{i}\right],
$$

we obtain, under the Gutzwiller approximation,

$$
\left\langle c_{i \sigma}^{\dagger} c_{j \sigma}\right\rangle \sim g_{t \sigma}(i) g_{t \sigma}(j) \chi_{i j \sigma},
$$

where

$$
\begin{gathered}
\chi_{i j \sigma}=\left\langle c_{i \sigma}^{\dagger} c_{j \sigma}\right\rangle_{0}, \\
g_{t \sigma}(i)=\left(1-r_{i \bar{\sigma}}\right) \sqrt{\frac{e_{i} q_{i}}{e_{i 0} q_{i 0}}}+r_{i \bar{\sigma}} \sqrt{\frac{q_{i} d_{i}}{q_{i 0} d_{i 0}}},
\end{gathered}
$$

where we used the relations derived previously for the fugacities $y_{i}, g_{i}$, and the various occupancies before and after projection. We collect all contributions to obtain

$$
\begin{aligned}
E= & -t \sum_{\langle i j\rangle \sigma} g_{t \sigma}(i) g_{t \sigma}(j)\left(\chi_{i j, \sigma}+\text { c.c. }\right) \\
& -\frac{3 J}{8} \sum_{\langle i j\rangle} g_{s}(i) g_{s}(j)\left(\chi_{i j}^{*} \chi_{i j}+\Delta_{i j}^{*} \Delta_{i j}\right) \\
& +J \sum_{\langle i j\rangle} g_{s}(i) g_{s}(j) m_{i} m_{j}+\sum_{i} U d_{i} .
\end{aligned}
$$

We note that the renormalization factors are formally identical to those obtained at zero temperature in Ref. 3. They reduce correctly to all known simple cases (at zero temperature) in the nonmagnetic ${ }^{1,2}$ or antiferromagnetic case. ${ }^{11,12}$ They are also formally similar to the results of the slaveboson formulation of the Hubbard model, ${ }^{13}$ but the entropy discussed below makes important differences.

\section{B. Entropy}

The second piece of the free energy is related to the entropy, which is, in principle, given by $S=-\operatorname{Tr}(\widetilde{\rho} / \tilde{Z}) \ln (\widetilde{\rho} / \widetilde{Z})$. Because the projection operator appears within the logarithm, a rigorous evaluation of the entropy is hopeless. In the following we first provide a lower bound of the exact entropy for the uniform nonmagnetic case, and then argue that the result can be extended immediately to the general unrestricted cases. For this purpose it turns out to be more convenient to work with $\rho$ in the canonical ensemble first. In this case, $S=-\operatorname{Tr}(\rho / Z) \ln (\rho / Z)$, where $Z=\operatorname{Tr} \rho$. We rewrite the projection operator as

$$
P=\sum E_{i_{1}} \ldots E_{i_{N_{E}}} Q_{j_{1}} \ldots Q_{j_{N_{Q}}} D_{k_{1}} \ldots D_{k_{N_{D}}},
$$

where $N_{E}, N_{Q}$, and $N_{D}$ are the total numbers of empty, singly occupied, and doubly occupied sites, respectively, in the system with $N$ sites, with $N=N_{E}+N_{Q}+N_{D}$. The summation is over the $N ! /\left(N_{E} ! N_{Q} ! N_{D} !\right)$ configurations of these sites. Clearly, the average empty, single, and double occupancies are $e=N_{E} / N, q=N_{Q} / N$, and $d=N_{D} / N$. The electron filling is given by $f=q+2 d$. Moreover, in the present form $P$ is a genuine projection operator satisfying $P^{2}=P$. Let us suppose that $\{|k\rangle\}$ are the eigenstates of the operator $\rho$ with the corresponding eigenvalues $\left\{\rho_{k}\right\}$. These states are clearly also the eigenstates of the projection operator $P$ with $P|k\rangle=|k\rangle$. Because of this, we have

$$
\rho_{k}=\langle k|\rho| k\rangle=\left\langle k\left|\rho_{0}\right| k\right\rangle .
$$

Consequently,

$$
\operatorname{Tr} \rho \ln \rho=\sum_{k} \rho_{k} \ln \rho_{k}=\sum_{k}\left\langle k\left|\rho_{0}\right| k\right\rangle \ln \left\langle k\left|\rho_{0}\right| k\right\rangle .
$$

Since $\rho_{0}$ is a positive definite Hermitian operator, we have the well-known inequality ${ }^{14,15}$

$$
\left\langle k\left|\rho_{0}\right| k\right\rangle \ln \left\langle k\left|\rho_{0}\right| k\right\rangle \leq\left\langle k\left|\rho_{0} \ln \rho_{0}\right| k\right\rangle
$$

so that

$$
\operatorname{Tr} \rho \ln \rho \leq \sum_{k}\left\langle k\left|\rho_{0} \ln \rho_{0}\right| k\right\rangle .
$$

We can remove the restriction of Hilbert space by bringing back the projection operator so that

$$
\operatorname{Tr} \rho \ln \rho \leq \operatorname{Tr} P\left(\rho_{0} \ln \rho_{0}\right) P .
$$

Now the projection operator is liberated from the logarithm. The total entropy is estimated as

$$
S=-\operatorname{Tr}(\rho / Z) \ln (\rho / Z) \geq \ln Z+\beta\left\langle P H_{0}\right\rangle_{0} /\langle P\rangle_{0},
$$

where we used $\ln \rho_{0}=-\beta H_{0}$ and $Z=\operatorname{Tr} \rho=\langle P\rangle_{0} Z_{0}$ with $Z_{0}=\operatorname{Tr} \rho_{0}$. Note that in the last inequality of the above equation, the projection operator stands only on one side of the Hamiltonian. The effect of this is quite different if $H_{0}$ is sandwiched between two projection operators. To see the effects of such a projection, we momentarily switch back to the grand-canonical ensemble. Since the projector $P$ has the effect of projecting out the desired empty, single, and double occupancies, in the grand-canonical ensemble we must replace $P$ by $\mathcal{P}^{2}$. In addition we must replace $\rho_{0}$ by $\widetilde{\rho}_{0}$ in doing the statistical average. For a hopping term in $H_{0}$, we have

$$
\begin{aligned}
\left\langle\mathcal{P}^{2} c_{i \sigma}^{\dagger} c_{j \sigma}\right\rangle_{0} /\left\langle\mathcal{P}^{2}\right\rangle_{0}= & \left\langle\left[n_{i \bar{\sigma}} y_{i}^{4} g_{i}^{2}+\left(1-n_{i \bar{\sigma}}\right) y_{i}^{2}\right]\right. \\
& \left.\times\left[n_{j \bar{\sigma}} y_{j}^{2}+\left(1-n_{j \bar{\sigma}}\right)\right] c_{i \sigma}^{\dagger} c_{j \sigma}\right\rangle_{0} /\left\langle\mathcal{P}_{i}^{2} \mathcal{P}_{j}^{2}\right\rangle_{0} \\
= & {\left.\left[r_{i \bar{\sigma}} d_{i} / d_{i 0}+\left(1-r_{i \bar{\sigma}}\right) q_{i} / q_{i 0}\right)\right] } \\
& \times\left[r_{j \bar{\sigma}} q_{j} / q_{j 0}+\left(1-r_{j \bar{\sigma}}\right) e_{j} / e_{j 0}\right]\left\langle c_{i \sigma}^{\dagger} c_{j \sigma}\right\rangle_{0} \\
= & \left\langle c_{i \sigma}^{\dagger} c_{j \sigma}\right\rangle_{0},
\end{aligned}
$$

where in the last step we used the fact that $f_{i}=q_{i}+2 d_{i}$, $e_{i}+q_{i}+d_{i}=1$, and $\left\langle n_{i \bar{\sigma}}\right\rangle_{0}=f_{i} / 2$ in a nonmagnetic state. The 
above equation means that projecting on one side does not lead to renormalization to the hopping terms under the Gutzwiller approximation. A similar relation holds for pairing terms. We conclude that in a nonmagnetic uniform state

$$
S \geq \ln Z_{0}+\beta\left\langle H_{0}\right\rangle_{0}+\ln \langle P\rangle_{0}=S_{0}+\Delta S,
$$

where

$$
S_{0}=-\sum_{n}\left[f_{n} \ln f_{n}+\left(1-f_{n}\right) \ln \left(1-f_{n}\right)\right]
$$

is the entropy of the unprojected free particle system and $\Delta S$ is the projection entropy $\Delta S=\ln \langle P\rangle_{0}$. Using the fact that under the Gutzwiller approximation

$$
\langle P\rangle_{0}=\frac{N !}{N_{E} ! N_{Q} ! N_{D} !} e_{0}^{N_{E}} q_{0}^{N_{Q}} d_{0}^{N_{D}},
$$

we obtain

$$
\Delta S=-N\left(e \ln \frac{e}{e_{0}}+q \ln \frac{q}{q_{0}}+d \ln \frac{d}{d_{0}}\right) .
$$

The above form of the entropy applies in nonmagnetic uniform states, where the canonical-ensemble projection operator satisfies $P^{2}=P$. We argue that the form of the entropy can be extended to general cases as follows. In the grand-canonical ensemble, $\tilde{Z}=\widetilde{Z}_{0}\left\langle\mathcal{P}^{2}\right\rangle_{0}=\widetilde{Z}_{0} \Pi_{i}{\widetilde{z_{i}}}_{\text {. The }}{\widetilde{z_{i}}}$ factors signify (artificial) degrees of freedom that are (superficially) independent of those in $\widetilde{Z}_{0}$. Within the factor $\widetilde{z_{i}}=e_{i 0}+y_{i}^{2} q_{i 0}+g_{i}^{2} y_{i}^{4} d_{i 0}$, the three "Boltzmann weights" are for empty, single, and double occupation states, being proportional to $e_{i}, q_{i}$, and $d_{i}$, respectively. Naively, this would amount to a sitewise entropy $-\left(e_{i} \ln e_{i}+q_{i} \ln q_{i}+d_{i} \ln d_{i}\right)$. However, there is a physical constrain that the entropy from $\tilde{z}_{i}$ should be upper bounded by the uncorrelated case since any correlation introduced by the projection reduces the active degrees of freedom. This constrain prescribes that the correct residual entropy caused by projection should be in the form of a comparison

$$
\Delta S=-\sum_{i}\left(e_{i} \ln \frac{e_{i}}{e_{i 0}}+q_{i} \ln \frac{q_{i}}{q_{i 0}}+d_{i} \ln \frac{d_{i}}{d_{i 0}}\right),
$$

which is negative definite, signifying the entropy loss due to projection. This form of $\Delta S$ may be viewed as a local-density approximation extension of the counterpart in the nonmagnetic uniform case. The estimation of the entropy is one of the central results of this work. We emphasize that the variational entropy gives a lower bound to the exact entropy, as desired to satisfy the Peierls theorem.

\section{Variation in the free energy and TRMFT}

We now have all ingredients to construct the free energy $F=E-T S$. We minimize it by cyclic two-step processes. First, we fix $\left\{r_{i \sigma}, g_{i}\right\}$ and vary $\psi_{n}$ and $f_{n}$ only. Second we modify $\left\{r_{i \sigma}, g_{i}\right\}$ subject to given total number of electrons. Since the renormalization factors $\left\{g_{t \sigma}(i), g_{s}(i)\right\}$ and the spin moments $\left\{m_{i}\right\}$ are fixed at the first stage, variation with respect to $\psi_{n}$ leads to a single-particle Shördinger-type equa- tion $h \psi_{n}=\epsilon_{n} \psi_{n}$ with $h$ a single-particle Hamiltonian. The variation with respect to $f_{n}$ merely yields a Fermi function $f_{n}=1 /\left(e^{\beta \epsilon_{n}}+1\right)$. This should self-consistently determine the order parameters $\chi_{i j}$ and $\Delta_{i j}$. The second-quantization form of $h$ is exactly $H_{0}$ we looked for in the first place and it reads

$$
\begin{aligned}
H_{0}= & -t \sum_{\langle i j\rangle \sigma} g_{t \sigma}(i) g_{t \sigma}(j)\left(C_{i \sigma}^{\dagger} C_{j \sigma}+\text { H.c. }\right) \\
& -\frac{3 J}{8} \sum_{\langle i j\rangle \sigma} g_{s}(i) g_{s}(j)\left(\chi_{i j}^{*} C_{i \sigma}^{\dagger} C_{j \sigma}+\text { H.c. }\right) \\
& -\frac{3 J}{8} \sum_{\langle i j\rangle \sigma \sigma^{\prime}} g_{s}(i) g_{s}(j)\left(\Delta_{i j}^{*} \epsilon_{\sigma \sigma^{\prime}} C_{j \sigma} C_{i \sigma^{\prime}}+\text { H.c. }\right) \\
& -\sum_{i \sigma} \mu_{i \sigma}\left(n_{i \sigma}-r_{i \sigma}\right),
\end{aligned}
$$

where $\epsilon_{\sigma \sigma^{\prime}}$ is the element of a $2 \times 2$ antisymmetric tensor and local Lagrangian parameters $\mu_{i \sigma}$ are introduced to fix the local densities $r_{i \sigma}$. The form of $H_{0}$ is similar to that derived at zero temperature. ${ }^{2,3}$ The reason is that under the Gutzwiller approximation the projection entropy is local, independently of the global structure of the single-particle states $\left\{\psi_{n}\right\}$.

At the second stage, we modify the parameters $\left\{r_{i \sigma}, g_{i}\right\}$ to further lower the free energy. In the restricted uniform case $r_{i \sigma}=r_{\sigma}$ and $g_{i}=g$. In the case of $t-J$ model, $g_{i}=0$ by definition.

Noticing that

$$
F_{0}=\left\langle H_{0}\right\rangle_{0}-T S_{0}=-T \ln \operatorname{Tr} e^{-\beta H_{0}}
$$

contains many parts of $E$, we can rewrite the physical free energy in a more convenient form

$$
F=\sum_{i} U d_{i}+J \sum_{\langle i j\rangle} g_{s}(i) g_{s}(j) m_{i} m_{j}-T \Delta S+F_{0} .
$$

Moreover, in the first variation stage, $\left\{r_{i \sigma}, d_{i}\right\}$, equivalent to $\left\{r_{i \sigma}, g_{i}\right\}$, completely specify $H_{0}$. Indeed, $q_{i}=f_{i}-2 d_{i}$, $e_{i}=1-d_{i}-q_{i}=1-f_{i}+d_{i}$. Combining $e_{i 0}=\left(1-r_{i \uparrow}\right)\left(1-r_{i \downarrow}\right)$, $d_{i 0}=r_{i \uparrow} r_{i \downarrow}$, and $q_{i 0}=f_{i}-2 d_{i 0}$, they determine all of the renormalization factors $\left(g_{t}\right.$ and $\left.g_{s}\right)$ in $H_{0}$, and self-consistency determines all of the order parameters and reproduces $r_{i \sigma}=\left\langle C_{i \sigma}^{\dagger} C_{i \sigma}\right\rangle_{0}$. Therefore, the two-step variational process is equivalent to a more practical process by varying $r_{i \sigma}, d_{i}$ combined with the self-consistency condition. Finally, the theory can be extended to the grand-canonical ensemble with the free energy $\Omega=F-\mu \sum_{i} f_{i}$ with a physical chemical potential $\mu$. In this case we have the liberty to vary $r_{i \sigma}$ without fixing the total electron number. We call this theory the TRMFT, in which the projection entropy $\Delta S$ is the key new ingredient.

At zero temperature, the effect of the entropy loss $\Delta S$ is absent in $F$, and we recover the zero-temperature RMFT. At finite temperatures, the system may choose to reduce the strength of the effective correlation by reducing the entropy loss and may eventually recover a free-particle theory at sufficiently high temperatures. This effect is absent in the slaveboson MF (SBMF) theory. The entropy loss also depends on the local charge and spin densities and will play a role in seeking inhomogeneous equilibrium states. 


\section{COMPARISON WITH GAUGE THEORIES FOR THE $t$ - $J$ MODEL}

Let us consider the uniform paramagnetic state of the $t-J$ model. In the SBMF theory, ${ }^{16}$ the electrons are decomposed into independent fermioninc spinons and bosonic holons. At high-temperature limit, one can neglect the band structure and a probability counting is sufficient to calculate the entropy. A site can be occupied by a spin-up spinon with probability $(1-x) / 2$ ( $x$ is the doping level beyond half filling), leading to an entropy per site

$$
s_{\uparrow}=-\frac{1-x}{2} \ln \frac{1-x}{2}-\frac{1+x}{2} \ln \frac{1+x}{2} .
$$

The spin-down spinons contributed equally, i.e., $s_{\downarrow}=s_{\uparrow}$. On the other hand, a site can be occupied by a holon with probability $x$, leading to the entropy contribution

$$
s_{h}=-x \ln x+(1+x) \ln (1+x) .
$$

The total entropy $S_{\mathrm{MF}}=S_{\uparrow}+S_{\downarrow}+S_{h}$ in the slave-boson meanfield theory in the high-temperature limit is therefore

$$
s_{\mathrm{MF}}=-x \ln x-(1-x) \ln (1-x)+2 \ln 2
$$

per site. This entropy is even larger than that of free electrons and is the reason why slave boson theory always predicts a paramagnetic insulating state at high temperatures, a state with unphysically high entropy. The missing ingredient in the mean-field theory is the gauge fluctuations, ${ }^{17}$ which acts to enforce the constrain that a site is always occupied by one and only one of the slave particles. Therefore, rather counterintuitively the (longitudinal) gauge degrees of freedom reduces, rather than increases the entropy due to the above effect. Indeed, the entropy contributed by them has been worked out to be $\mathrm{b}^{18}$

$$
s_{g}=-(1+x) \ln 2 .
$$

The total entropy is therefore given by

$$
s=s_{\mathrm{MF}}+s_{g}=-x \ln x-(1-x) \ln \frac{1-x}{2},
$$

which reflects exactly the fact that a site is empty, occupied by a spin-up or spin-down electron with probabilities $x,(1-x) / 2$, and $(1-x) / 2$, respectively.

Now let us look at the same problem from the TRMFT point of view. Here no holons are involved. The entropy from the free fermions in the mean-field theory in the hightemperature limit is given by

$$
s_{0}=-(1-x) \ln \frac{1-x}{2}-(1+x) \ln \frac{1+x}{2},
$$

which is the fermionic piece of $S_{\mathrm{MF}}$ in the above. To calculate the projection entropy, we recall that $e=x$ and $e_{0}=(1+x)^{2} / 4, q=1-x$ and $q_{0}=\left(1-x^{2}\right) / 2, d=0$ and $d_{0}=(1-x)^{2} / 4$. The projection entropy is

$$
\Delta s=-x \ln \frac{4 x}{(1+x)^{2}}-(1-x) \ln \frac{2}{1+x},
$$

which is indeed negative definite. Combining $s_{0}$ and $\Delta s$ yields exactly the same expression for $s$ as above. We therefore see that at the TRMFT level the entropy is already lower than that of SBMF, and the projection entropy worked out in this paper further improves to give the exact entropy in the high-temperature limit.

\section{DOUBLE OCCUPANCY ON THE ANDERSON IMPURITY}

As the first simple but nontrivial application, we consider an Anderson impurity coupled to a conduction band. The Hamiltonian is given by $H=H_{f}+H_{c}+H_{V}$, where $H_{f}$ $=\Sigma_{\sigma}\left(\varepsilon_{f}-\mu\right) f_{\sigma}^{\dagger} f_{\sigma}+U n_{f \uparrow} n_{f \downarrow}$ describes the impurity atom in insolation, $H_{c}=\sum_{\mathbf{k} \sigma}\left(\varepsilon_{\mathbf{k}}-\mu\right) c_{\mathbf{k} \sigma}^{\dagger} c_{\mathbf{k} \sigma}$ describes the conduction electrons (in momentum space), and $H_{t}=-t \Sigma_{\sigma}\left(f_{\sigma}^{\dagger} c_{0 \sigma}+\right.$ H.c. $)$ describes the hybridization between the impurity and the conduction electrons at a real-space position 0 . For the simplest purpose, we will consider $\varepsilon_{f}=-U / 2$ and assume that $\varepsilon_{\mathbf{k}}$ is symmetric about zero. In this case $\mu=0$ guarantees that the system is half filled everywhere by strict particle-hole symmetry. Since only the impurity electrons interact, we only have to renormalize the impurity-related part of the Hamiltonian. On the impurity site, we have $e=d, q+2 d=1$, $e_{0}=d_{0}=1 / 4$, and $q_{0}=1 / 2$. According to the above TRMFT, we can take $d$ as the only variational parameter and write $H_{0}=-g_{t} H_{t}+H_{c}$ as the renormalized free Hamiltonian with $g_{t}=\sqrt{8 d(1-2 d)}$. The free energy is given by

$$
\begin{aligned}
F= & \varepsilon_{f}+U d-T \Delta S-T \ln \operatorname{Tr} e^{-\beta H_{0}} \\
= & \varepsilon_{f}+U d+T[2 d \ln (2 d)+(1-2 d) \ln (1-2 d)+\ln 2] \\
& -2 T \operatorname{Tr} \ln G_{f}^{-1}+F_{c} .
\end{aligned}
$$

In the last equality the factor of 2 before the trace symbol accounts for spin species, $G_{f}=1 /\left[i \omega_{n}-g_{t}^{2} t^{2} g_{c}\right]$ is the impurity Green's function, $F_{c}$, and $g_{c}=\Sigma_{\mathbf{k}} 1 /\left(i \omega_{n}-\varepsilon_{\mathbf{k}}\right)$ (normalized to unit volume) is the free energy and local Green's function of the conduction band when it is uncoupled to the impurity. In arriving at the above result we have performed path integrals by part. Varying $F$ with respect to $d$, we have

$$
U=-2 T \ln \frac{2 d}{1-2 d}-16(1-4 d) T \sum_{n}\left(t^{2} g_{c} G_{f}\right)
$$

This result is exact both in the atomic limit $t=0$ [where $\left.d=1 /\left(2+2 e^{U / 2 T}\right)\right]$ and in the interactionless limit $U=0$ (where $d=1 / 4$ ). In general cases, the above equation yields a monotonic function $U$ in terms of $d$ at a given temperature $T$, and it can be easily inverted numerically to find $d$ as a function of $U$ and $T$. To proceed, we assume a rectangular density of states (DOS) for the conduction band, $N_{c}(\omega)=\theta(1-|\omega|) / 2$ (with a full bandwidth $W=2$ ) and write $g_{c}=\int d \omega N_{c}(\omega) /\left(i \omega_{n}-\omega\right)$. We further take $\Gamma=\pi N_{c}(0) t^{2}=W / 4$ for definiteness. The temperature dependence of $d$ for $U=2,4$ are presented in Fig. 1 (solid lines) and is compared to the corresponding NRG results (symbols). We see perfect 


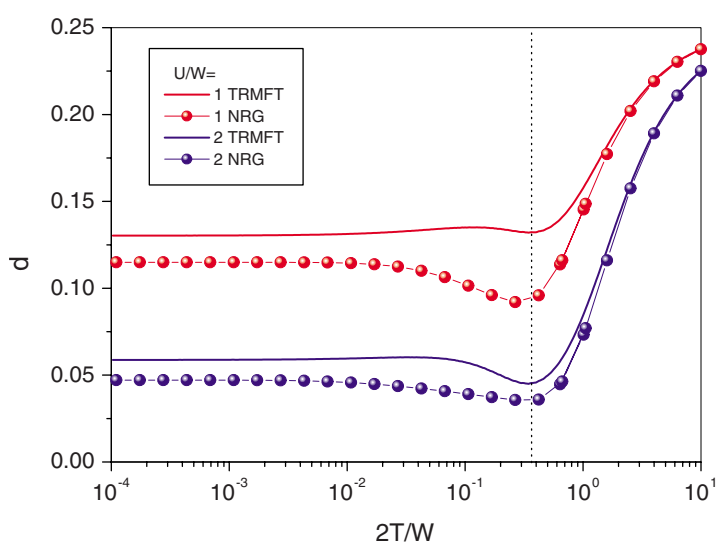

FIG. 1. (Color online) Temperature dependence of the double occupancy in the Anderson impurity model for $U / W=1$ and $U / W$ $=2$. Solid lines (symbols) are obtained by TRMFT $(\mathrm{NRG})$. The dashed line highlights the dip around the crossover temperature.

or qualitative agreement in the three temperature regimes: the activation regime $T \sim W$ in which $\ln d \sim-U / 2 T$; the local moment regime in which $d$ dips toward zero (highlighted by the dashed line), and the Kondo regime $T<T_{K}$ where $d$ saturates. The Kondo temperature $T_{K}$ from the Bethe ansatz solution is ${ }^{19} T_{K}=\sqrt{U \Gamma / 2} \exp (-\pi U / 8 \Gamma)$. We find $2 T_{K} / W$ $=0.147$ (or 0.043 ) for $U / W=1$ (or 2). We have therefore reached temperatures far below $T_{K}$ in Fig. 1 . On the other hand, we naturally get the free atomic limit $d \rightarrow 1 / 4$ for $T \gg W, U$.

\section{METAL-INSULATOR TRANSITION IN THE HALF- FILLED HUBBARD MODEL}

The Hubbard model at half filling can display a transition from a metal to an insulator as the control parameter $U / W$ varies, where $W$ is the bandwidth. At finite temperature the thermal-energy scale $T$ enters. We consider the uniform paramagnetic state characterized by a bare band DOS $N(\omega)$ $=(2 / \pi) \sqrt{1-\omega^{2}}$ (suitable for a Bethe lattice) with a full bandwidth $W=2$. At half filling we again have $e=d, q=1-2 d$, $e_{0}=d_{0}=1 / 4$, and $q_{0}=1 / 2$. According to the TRMFT, all hopping integrals are renormalized by $g=g_{t}^{2}=8 d(1-2 d)$ so is the band energy. Therefore the free-energy density per site is given by

$$
\begin{aligned}
f= & U d+T[2 d \ln 2 d+(1-2 d) \ln (1-2 d)+\ln 2] \\
& -2 T \int d \omega N(\omega) \ln \left(1+e^{-\beta g \omega}\right) .
\end{aligned}
$$

Variation with respect to $d$ yields

$$
U=-2 T \ln \frac{2 d}{1-2 d}-16(1-4 d) \int \frac{d \omega N(\omega) \omega}{1+e^{\beta g \omega}} .
$$

The double occupancy $d$ as a function of $T$ is presented in Fig. 2 (solid lines) for $U=1.3 W n / 4(n=0,1, \ldots, 8)$. The double-head arrow specifies the TRMFT result for $U=1.3 \mathrm{~W}$, which is compared to the corresponding result obtained by DMFT using NRG as the impurity solver (symbols). ${ }^{20} \mathrm{We}$

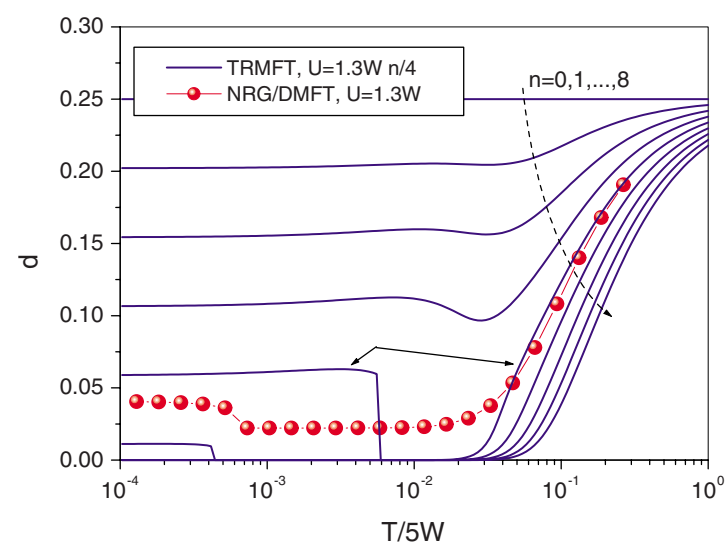

FIG. 2. (Color online) Temperature dependence of the double occupancy in a Hubbard model. The solid lines are obtained by TRMFT for $U=1.3 W n / 4(n=0,1, \ldots, 8)$. The double-head arrow specifies the TRMFT result for $U=1.3 W$, which is compared to the corresponding result obtained by DMFT using NRG as the impurity solver (symbols).

see qualitative agreement. Moreover, we see from the TRMFT curves that for $U<W$ the double occupancy develops a dip around a crossover temperature. By comparison with the Anderson impurity problem discussed above, we understand the dip as indicating the tendency of the formation of local moment. The crossover temperatures are presented in Fig. 3 (dotted line). As $U$ increases further, the dip evolves to a finite-temperature window within which $d \rightarrow 0$ in the TRMFT result. This is a signature of a saturated local moment. We assign the lower ends of these windows as the boundary of the first-order phase transition between the lowtemperature paramagnetic metallic phase and the higher temperature paramagnetic insulating phase. The phase boundary is plotted in Fig. 3 (solid line). There is a finite jump of $d$ across the boundary, except at the zero-temperature end (triangle) and the end point at roughly $T=0.11 \mathrm{~W}$ (square). The critical $U$ for the phase transition at $T=0$ (triangle) is slightly above that within DMFT, and the starting temperature of the crossover line (square) is about three or four times larger than that in DMFT. Apart from such quantitative discrepancy, the phase diagram in Fig. 3 is in good agreement with the single-site DMFT results. ${ }^{21,22}$

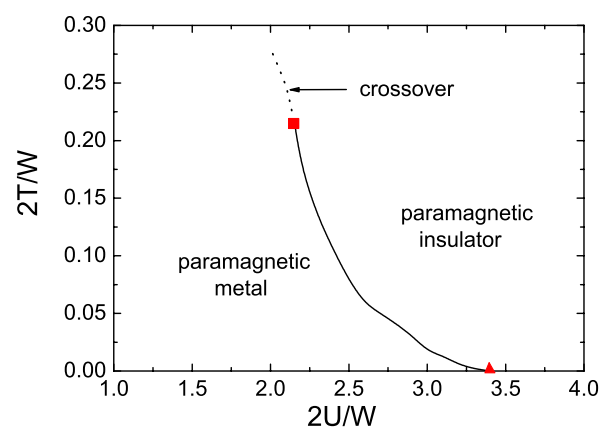

FIG. 3. (Color online) Phase diagram of the half-filled Hubbard model. The transition line is first order at finite temperatures and it terminates at two second-order transition points at zero temperature (triangle) and at a finite temperature about $T=0.11 \mathrm{~W}$ (square). The dotted line indicates the crossover. 


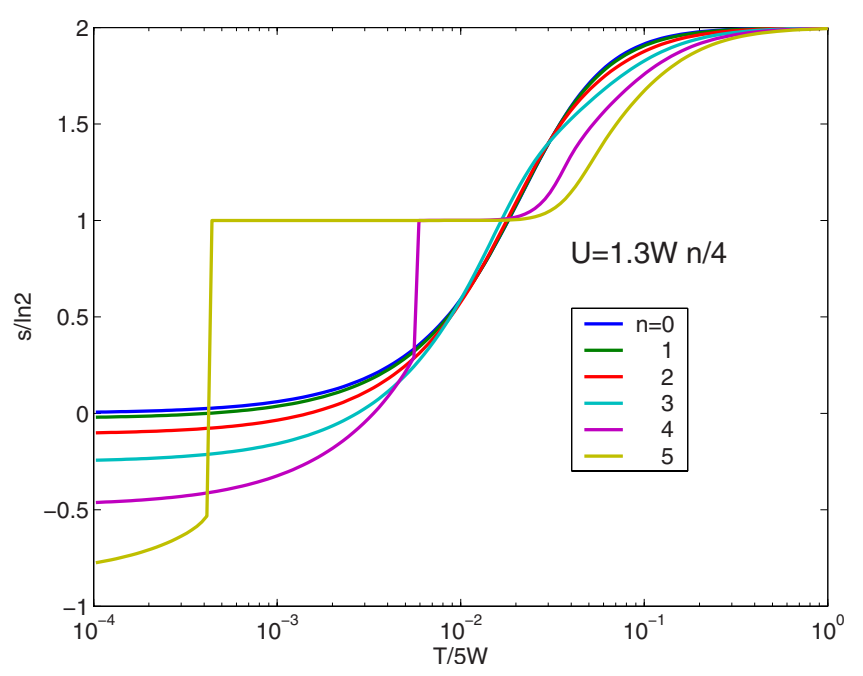

FIG. 4. (Color online) Temperature dependence of the entropy per site in a Hubbard model obtained by TRMFT for $U=1.3 \mathrm{Wn} / 4$ $(n=0,1, \ldots, 5)$.

In order to understand the phase diagram better, we plot the entropy as a function of temperature in Fig. 4 for $U=1.3 \mathrm{Wn} / 4 \quad(n=0,1,2, \ldots, 5)$. In the high-temperature limit, all curves approach $s=2 \ln 2$, as desired in the free atomic limit (at half filling). The curves are continuous for $n \leq 3$ but develop plateaus with $s=\ln 2$ for $n \geq 4$, followed by downward jumps with decreasing temperature. The plateau corresponds to $d=0$. Here the bandwidth becomes zero so that the free-fermion entropy is $s_{0}=2 \ln 2$ while the projection entropy is $\Delta s=-\ln 2$, yielding a total entropy $s=\ln 2$. Without the projection entropy $s$ would be already saturated so that $d$ would have to remain zero at higher temperatures, leading to phase transitions for any $U>0$ (not shown). With the projection entropy, the plateau value is exactly that of a local moment while the entropy jump defines the first-order phase transition in Fig. 3. There is a caveat in TRMFT, however. The entropy becomes negative in the zero-temperature limit for $U>0$. This is because here the free-fermion entropy approaches zero while the projection entropy is negative for $d<d_{0}$. The negative entropy is, in principle, unphysical and indicates the overestimation of the projection entropy (in magnitude) at low temperatures. However, it is allowed in a variational theory. After all, at low enough temperatures the entropy does not play an important role.

\section{SUMMARY AND REMARKS}

We generalized the Gutzwiller projectional variational method for the ground state for strongly correlated electron systems to the case of finite temperature. Under the Gutzwiller approximation, we show that this maps to a finite temperature renormalized mean-field theory, with the projection entropy as the important ingredient. We illustrate the application of the theory to the Anderson impurity problem and the half-filled Hubbard model and compare the theory to more elaborate techniques. We find qualitatively good agreement. The method is variational (thus nonperturbative), unrestricted and operates at any finite temperatures, and can be applied to a wide variety of Hubbard, $t-J$, and Anderson impurity models.

A few remarks are in order before closing: (1) a phenomenological finite-temperature theory under the Gutzwiller approximation was proposed for a nearly localized Fermi liquid. $^{23}$ In this theory a weighting function for the momentum-dependent entropy contributions was introduced to reflect the effects of projection. In another phenomenological theory, ${ }^{24}$ the projected free energy is assumed to that of the free system subject to a renormalization factor common to both the kinetic energy and the entropy. It would be interesting to see whether these prescriptions satisfy the variational requirement for the entropy. It would also be interesting to see how such approaches can be extended to inhomogeneous systems. In comparison, the present theory follows "microscopically" from the variational principle for unrestricted states. (2) The projection entropy in our case depends on local occupancies (for the empty, singly occupied, and doubly occupied states) and is therefore important in seeking inhomogeneous states. However, it is insensitive to magnetic or superconducting orders. A possible improvement is to extend the estimation of the projection entropy to be order-parameter sensitive also. (3) In the insulating paramagnetic state of the half-filled Hubbard model, the entropy is $\ln 2$ per site in the TRMFT, implying a completely uncorrelated system with local moments. In reality, the local moments can develop spatial/temporal correlations even in the insulating state. This physics could be picked up by adding the spin-exchange interaction to the standard Hubbard model and the TRMFT (see Sec. II C). The phase diagram in this situation could be improved but is left as a further study. (4) Apart from band renormalization and static order parameters, the TRMFT also yields information on dynamical properties of the system. As in the zero-temperature $\mathrm{RMFT}^{3}{ }^{3}$ the variation in the internal energy $\delta E$ versus that of the eigenorbital occupancy $\delta f_{n}$ satisfies $\delta E=\sum_{n} \epsilon_{n} \delta f_{n}$ to leading order. Therefore the single-particle energy $\epsilon_{n}$ of $H_{0}$ describes consistently the pole of quasiparticle excitations in the sense of Landau Fermi liquid. On the other hand, the factor $g_{t \sigma}(i)$ measures the overlap between the bare quasiparticle state in the TRMFT and a corresponding real electron wave function at the given site. Therefore, the electron Green's function may be written as $G(i \sigma, j \sigma)=g_{t \sigma}(i) g_{t \sigma}(j) G_{0}(i \sigma, j \sigma)+G_{i n c}(i \sigma, j \sigma)$, where the first contribution is the coherent part with $G_{0}$ obtained from $H_{0}$. The incoherent part $G_{i n c}$ is not available at the mean-field level. On the other hand, static response functions can be obtained by TRMFT subject to suitable applied fields. Furthermore, a bare bubble calculation using $G$ could provide useful insights into dynamic two-particle response functions, although caution must be paid to vertex corrections.

\section{ACKNOWLEDGMENTS}

Q.H.W. thanks T. Pruschke for fruitful discussions and providing us DMFT results for the Hubbard model. F.C.Z. thanks T. M. Rice for many helpful discussions. The work in Nanjing was supported by NSFC under Grants No. 10974086 and No. 10734120, the Ministry of Science and 
Technology of China under Grants No. 2006CB921802 and No. 2006CB601002, and the 111 Project under Grant No.
B07026. The work in Hong Kong was supported by the RGC grants of Hong Kong. *qhwang@nju.edu.cn

${ }^{1}$ D. Vollhardt, Rev. Mod. Phys. 56, 99 (1984).

${ }^{2}$ F. C. Zhang, C. Gros, T. M. Rice, and H. Shiba, Supercond. Sci. Technol. 1, 36 (1988).

${ }^{3}$ Q. H. Wang, Z. D. Wang, Y. Chen, and F. C. Zhang, Phys. Rev. B 73, 092507 (2006).

${ }^{4}$ P. W. Anderson, P. A. Lee, M. Randeria, T. M. Rice, N. Trivedi, and F. C. Zhang, J. Phys.: Condens. Matter 16, R755 (2004).

${ }^{5}$ C. Gros, R. Joynt, and T. M. Rice, Phys. Rev. B 36, 381 (1987); C. Gros, Ann. Phys. (N.Y.) 189, 53 (1989); H. Yokoyama and H. Shiba, J. Phys. Soc. Jpn. 57, 2482 (1988); T. K. Lee and S. Feng, Phys. Rev. B 41, 11110 (1990).

${ }^{6}$ See, e.g., F. C. Zhang, Phys. Rev. Lett. 90, 207002 (2003); J. Y. Gan, Y. Chen, Z. B. Su, and F. C. Zhang, ibid. 94, 067005 (2005).

${ }^{7}$ R. Peierls, Phys. Rev. 54, 918 (1938).

${ }^{8}$ B. A. Bernevig, R. B. Laughlin, and D. I. Santiago, Phys. Rev. Lett. 91, 147003 (2003); R. B. Laughlin, Philos. Mag. 86, 1165 (2006).

${ }^{9}$ P. W. Anderson and N. P. Ong, J. Phys. Chem. Solids 67, 1 (2006).

${ }^{10}$ B. Edegger, N. Fukushima, C. Gros, and V. N. Muthukumar, Phys. Rev. B 72, 134504 (2005).
${ }^{11}$ H. X. Huang, Y. Q. Li, and F. C. Zhang, Phys. Rev. B 71, 184514 (2005).

${ }^{12}$ M. Ogata and A. Himeda, J. Phys. Soc. Jpn. 72, 374 (2003).

${ }^{13}$ G. Kotliar and A. E. Ruckenstein, Phys. Rev. Lett. 57, 1362 (1986).

${ }^{14}$ R. G. Parr and W. Yang, Density-Functional Theory of Atoms and Molecules (Clarendon, Oxford, 1989).

${ }^{15}$ K. Tanabe and H. Nakada, Phys. Rev. C 71, 024314 (2005).

${ }^{16}$ G. Kotliar and J. Liu, Phys. Rev. B 38, 5142 (1988).

${ }^{17}$ D. H. Lee, Phys. Rev. Lett. 84, 2694 (2000); Q. H. Wang, ibid. 92, 057003 (2004).

${ }^{18}$ R. Hlubina, W. O. Putikka, T. M. Rice, and D. V. Khveshchenko, Phys. Rev. B 46, 11224 (1992).

${ }^{19}$ See, e.g., A. C. Hewson, The Kondo Problem to Heavy Fermions (Cambridge University Press, Cambridge, UK, 1993).

${ }^{20}$ T. Pruschke (private communication).

${ }^{21}$ A. Georges, G. Kotliar, W. Krauth, and M. J. Rozenberg, Rev. Mod. Phys. 68, 13 (1996), and references therein.

${ }^{22}$ J. Joo and V. Oudovenko, Phys. Rev. B 64, 193102 (2001).

${ }^{23}$ K. Seiler, C. Gros, T. M. Rice, K. Ueda, and D. Vollhardt, J. Low Temp. Phys. 64, 195 (1986).

${ }^{24}$ K. A. Chao and K.-F. Berggren, Phys. Rev. B 15, 1656 (1977); Y. B. Kudasov, Phys. Lett. A 193, 315 (1994). 